estimates ranged from $0.86-1.25$ injuries/dance teacher. Due to limited investigation of risk factors, results could not be stratified by sex, age, or dance style.

Conclusions There have been few primary investigations of injury in dance education settings, despite large rates of dance participation. Consistent injury and exposure definitions, and high-quality prospective studies are needed for examining injury risk in the dance teaching environment.

\section{ASSOCIATION BETWEEN BASELINE FACTORS AND RISK OF INJURY AMONGST PRE-PROFESSIONAL DANCERS}

1,2,35arah J Kenny, ${ }^{1} \mathrm{KV}$ Vineetha Warriya, ${ }^{1,4}$ Luz Palacios-Derflingher, ${ }^{5}$ Jackie Whittaker, 1,2,6 Carolyn Emery, 'Meghan Critchley. 'Sport Injury Prevention Centre, Faculty of KinesiologyUniversity of Calgary, Calgary, Canada; ${ }^{2}$ Alberta Children's Hospital Research Institute, University of Calgary, Calgary, Canada; ${ }^{3} O^{\prime} B r i e n$ Institute for Public Health, University of Calgary, Calgary, Canada; ${ }^{4}$ Department of Pediatrics, Cumming School of Medicine, University of Calgary, Calgary, Canada; ${ }^{5}$ Department of Physical Therapy, Faculty of Medicine, University of British Columbia, Vancouver, Canada; ${ }^{6}$ Department of Community Health Sciences, Cumming School of Medicine, University of Calgary, Calgary, Canada

10.1136/bjsports-2021-IOC.118

Background Few investigations utilize evidence-informed preparticipation evaluation, inclusive injury definitions, and prospective surveillance to identify risk factors for dance-related injury.

Objective To identify baseline injury risk factors that may be associated with dance-related musculoskeletal (MSK) complaints in pre-professional dancers.

Design Prospective cohort study.

Setting Pre-professional ballet school; university dance program.

Participants Dancers registered in full-time ballet $[n=85,77$ females, median (range) age 15 years (11-19)] and contemporary $[n=60,58$ females, 19 years $(17-30)]$ training.

Assessment of Risk Factors Pre-participation evaluation comprised of one-year injury history (yes/no), previous training (years), Athletic Coping Skills Inventory-28 (ACSI;score), body mass index $\left(\mathrm{BMI} ; \mathrm{kg} / \mathrm{m}^{2}\right)$, total bone mineral density $\left(\mathrm{g} / \mathrm{cm}^{2}\right)$, ankle plantar/dorsiflexion (degrees), active standing turnout (degrees), three lumbopelvic control tasks (high/low risk), unipedal dynamic balance (seconds), Y-Balance Test ( $\mathrm{cm})$. Weekly dance hours were self-reported throughout one academic year. Main Outcome Measurements Self-reported MSK complaints (any physical complaint leading to difficulties participating in dance, regardless of consequences) were captured weekly by online modified Oslo Sports Trauma Research Centre's Questionnaire on Health Problems during academic year. MSK complaints were recorded (yes/no) for each participant for each week.

Results Response rate was 99\%, with 81\% (117/145) of dancers reporting at least one MSK complaint. Of the 1521 complaints (19\% first-time, $81 \%$ recurrent), ankle (22\%), knee $(21 \%)$, and foot $(12 \%)$ accounted for majority. Potential factors were identified through systematic review and a generalized linear mixed model was used to analyze the binary outcome measure. Injury history [Odds Ratio (OR) 7.37; 95\% CI $(3.41,15.91)]$ and previous week's dance hours [OR 1.02; $95 \%$ CI $(1.01,1.03)]$ were significantly associated with MSK complaint.

Conclusions Prevalence of MSK complaints amongst pre-professional ballet and contemporary dancers is high and significantly associated with injury history and training volume.
Future studies implementing injury prevention should incorporate training load monitoring to address the dynamic, recursive nature of dance injury etiology.

\section{ABSTRACT WITHDRAWN}

\section{MUSCULOSKELETAL INJURIES AND DANCE EXPOSURE ACROSS THREE YEARS IN ELITE ADOLESCENT BALLET DANCERS: IS THERE A PATTERN?}

'Valeriya Volkova, 1,2,3 Sarah J Kenny. 'Sport Injury Prevention Research Centre, Faculty of Kinesiology, University of Calgary, Calgary, Canada; ${ }^{2}$ Alberta Children's Hospital Research Institute, University of Calgary, Calgary, Canada; ${ }^{3} \mathrm{O}^{\prime}$ Brien Institute for Public Health, University of Calgary, Calgary, Canada

\subsection{6/bjsports-2021-IOC.119}

Background Dance is a popular activity among youth at all levels and involves large volumes of training. Reported injury estimates are high in this population, warranting investigation of training volume and injury patterns throughout multiple training years.

Objective To describe musculoskeletal (MSK) injuries and dance exposure in elite adolescent ballet dancers across three training seasons.

Design Prospective cohort.

Setting Vocational ballet school.

Patients (or Participants) Dancers registered in full-time elitelevel ballet training $[\mathrm{n}=172 ; 152$ female, median (range) age 15 years $(10-21)]$.

Interventions (or Assessment of Risk Factors) Volume of dance training (hours) was self-reported weekly via online questionnaire.

Main Outcome Measurements Dance-related MSK injuries were registered by self-report (weekly, online modified Oslo Sports Trauma Research Centre Questionnaire on Health Problems). Three injury definitions were utilized: physical complaints (any complaint leading to difficulty participating), medical attention (any complaint resulting in care from a clinician), time-loss (unable to complete $\geq 1$ class/rehearsal/performance for $\geq 1$ day(s)). Means (SD) for weekly dance hours and weekly injury prevalence (proportion of dancers injured/week) were estimated.

Results Questionnaire response rates ranged from 89\% (year 3) to $99 \%$ (year 1). Across three training years, dance volume ranged from 19.26 hours/week (SD:4.59) (year 3) to 22.17 (SD:5.28) (year 2), mean weekly injury prevalence ranged from $21 \%$ (year 3) to $31 \%$ (year 1) (physical complaints); $15 \%$ (years $2 \& 3$ ) to $24 \%$ (year 1) (medical attention), and $5 \%$ (years 1\&3) to $9 \%$ (year 2) (time-loss). Weekly selfreported injuries mirrored the fluctuations in weekly dance exposure across the three years.

Conclusions Elite adolescent dancers report long hours of weekly training with variations across a training season. When defined as a physical complaint, dance-related MSK injury estimates are high in this population. As dance exposure hours increase, so do self-reported injury estimates. Further research is needed to explore associations between intensity of dance exposure (i.e., training load) and injuries to inform development of effective injury prevention strategies for this high-risk group of artistic athletes. 